\title{
Symplectic Integration for Complex Wigglers
}

\author{
Étienne Forest*and Kazuhito Ohmi \\ KEK, National Laboratory for High Energy Physics, \\ Tsukuba, Ibaraki 305, Japan
}

19 August 1992

\begin{abstract}
Using the example of the helical wiggler proposed for the KEK photon factory, we show how to integrate the equation of motion through the wiggler. The integration is performed in cartesian coordinates. For the usual expanded Hamiltonian (without square root), we derive a first order symplectic integrator for the purpose of tracking through a wiggler in a ring. We also show how to include classical radiation for the computation of the damping decrement.
\end{abstract}

\footnotetext{
"Permanent address, Exploratory Studies Group, Accelerator and Research Division, Lawrence Berkeley Laboratory, 1 Cyclotron Road, MS 71-259, Berkeley, California 94720, USA.
} 


\section{Contents}

1 Introduction $\quad 2$

2 Exact Equation of Motion 3

2.1 Exact Hamiltonian . . . . . . . . . . . . . . . . . . . 3

2.2 Integration Method . . . . . . . . . . . . . . . . 4

3 Symplectic Methods on the Approximate Hamiltonian 5

4 Discussion 6

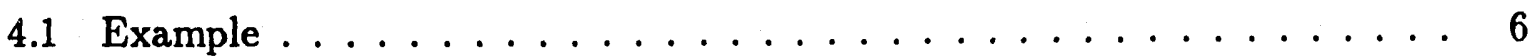

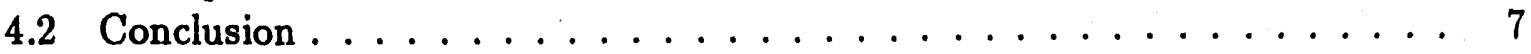

\section{Introduction}

No Need for the Closed Orbit In the field of accelerator design, the inclusion of wiggler and undulator in a tracking code has always looked like a special task. We believe that the difficulties are more imaginary than real. In fact, plain integration through a wiggler is very simple because the fields are always simply expressed in cartesian coordinates. The starting point are the famous Halbach formulas[1]. For the KEK photon factory, we can approximate the helical wiggler by the following expressions for the vector potential:

$$
\begin{aligned}
f \equiv \frac{A_{x}}{p_{0} / q} & =\frac{F_{0}}{k} \cos \left(k_{1} x\right) \cosh \left(k_{2} y\right) \sin (k z)-\frac{k_{4} G_{0}}{k k_{3}} \sinh \left(k_{3} x\right) \sin \left(k_{4} y\right) \sin (k z+\phi) \\
g \equiv \frac{A_{y}}{p_{0} / q} & =\frac{F_{0} k_{1}}{k k_{2}} \sin \left(k_{1} x\right) \sinh \left(k_{2} y\right) \sin (k z)-\frac{G_{0}}{k} \cosh \left(k_{3} x\right) \cos \left(k_{4} y\right) \sin (k z+\phi) \\
A_{z} & =0
\end{aligned}
$$

$$
\text { where } k_{2}^{2}=k^{2}+k_{1}^{2} \quad k_{3}^{2}=k^{2}+k_{4}^{2}
$$

$q$ and $p_{0}$ are the charge and the reference momentum of tracked particles. $F_{0}, G_{0}, k$ 's and $\phi$ are parameters characterizing the wiggler.

The expression of Eq.(1) assumes an infinitely long wiggler of period $\lambda=\frac{2 \pi}{k}$ along the $z$-direction. Therefore it is simplest to integrate it using the normal cartesian coordinates where $z$ parametrizes the motion (time-like variables).

Previous works[2] in the field are based on L.Smith's Hamiltonian [3] which is written in terms of deviation from a "closed orbit" through the wiggler and, to compound the problem, use a complicated Frenet-Serret system. If we consider that our purpose is to obtain the one t'irn map ( tune and beta function etc...) and is to track particles in a ring with a computer, this choice of the coordinate system will complicate our problem for many reasons: 
1. the computer does not care about orbit distortions due to the linear part of the Hamiltonian

2. we do not know the closed orbit until we close the ring

3. the wiggler field is horrible in these variables

4. the electron does not care about the frame used: so use the simplest

The Hamiltonian in terms of deviation from a "closed orbit" start with second order terms and may be suitable to treat an oscillation around the closed orbit analytically (although this is even questionable in complex cases). But writing a tracking code in terms of Frenet-Serret coordinate around an actual wiggler orbit will not be suitable. If we have the ability to compute maps, we can match wigglers and compute all global quantities desired. Maps are always extractable thanks to automatic differentiation. For a general discussion of the principles behind modern tracking and analysis in circular rings, the reader should consult[4].

\section{Exact Equation of Motion}

\subsection{Exact Hamiltonian}

If we assume that the particle is ultra-relativistic, the exact Hamiltonian for the helical wiggler is given in cartesian variables by:

$$
\begin{aligned}
H= & -\sqrt{\left(1+p_{-\ell}\right)^{2}-\left(p_{x}-f\right)^{2}-\left(p_{y}-g\right)^{2}}-\epsilon p_{-\ell} \\
& \epsilon=0 \rightarrow \text { total path length computed } \\
& \epsilon=1 \rightarrow \text { differential path length computed. }
\end{aligned}
$$

where $f$ and $g$ are given in Eq.(1).

In Eq.(2), the differential path length is the total path length minus the length of the wiggler. The 6 -vector describing the motion of a particle is

$$
x=\left(x, p_{x}, y, p_{y},-\ell, p_{-\ell}\right)
$$

where $-\ell$ and $p_{-\ell}$ are the negative path length and its conjugate momentum which is equivalent to the momentum deviation $\delta p / p_{0}$, respectively.

The equations of motion are given by

$$
\begin{array}{rlrl}
\frac{d x}{d z} & =[x, H] \\
& \downarrow \\
\frac{d x}{d z} & =\frac{\partial H}{\partial p_{x}} & \frac{d p_{x}}{d z}=-\frac{\partial H}{\partial x} \\
\frac{d y}{d z} & =\frac{\partial H}{\partial p_{y}} & \frac{d p_{y}}{d z}=-\frac{\partial H}{\partial y} \\
\frac{d(-\ell)}{d z} & =\frac{\partial H}{\partial p_{-\ell}} & \frac{d p_{-\ell}}{d z}=0 .
\end{array}
$$


In the case of the beam envelope calculation it may be necessary to add radiation to the equation. The result for the deterministic part of the motion is:

$$
\begin{aligned}
\frac{d x}{d z} & =[x, H]+r \\
r_{6} & =-K_{\text {rad }}\left(1+p_{-\ell}\right)^{2}\left|\frac{q B_{\perp}}{p_{0}}\right|^{2} \frac{\partial H_{c=1}}{\partial p_{-\ell}} \\
r_{2} & =\frac{p_{x} r_{6}}{1+p_{-\ell}} \quad r_{4}=\frac{p_{y} r_{6}}{1+p_{-\ell}}
\end{aligned}
$$

The constant $K_{\text {rad }}$ is given for example by Sands[5] and has the value $1.40789357 \times$ $10^{-5} E_{0}^{3}$ where $E_{0}$ is the reference energy expressed in $\mathrm{GeV}$. The $\left|B_{\perp}\right|$ is the modulus of the component of the magnetic field perpendicular to the direction of propagation. The direction of propagation is obtained using

$$
\begin{gathered}
\mathbf{e}=\frac{1}{\sqrt{1+\frac{d x^{2}}{d z}+\frac{d y^{2}}{d z}}\left(\frac{d x}{d z}, \frac{d y}{d z}, 1\right)} \\
\left|\mathbf{B}_{\perp}\right|=|\mathbf{B} \times \mathbf{e}|,
\end{gathered}
$$

and, of course the magnetic field $\mathbf{B}$ is obtained from $\nabla \times \mathbf{A}$. The non zero value for $r_{2}$ and $r_{4}$ in Eq.(5) is an expression of the conservation of the unit vector e during the radiation process.

The stochastic part of the beam envelope calculation can be done in a similar manner.

\subsection{Integration Method}

This paper would stop here if the non-radiative part of Eq.(5) (i.e. Eq.(4)) was amenable to explicit symplectic integration. Why do we say so? If you have a symplectic integrator, then the criteria which must be used in deciding the number of steps of integration are not different from those used in the case of a quadrupole or sextupole. The number of thin lenses (i.e. integration steps) used for a sextupole depends only on some global properties like dynamic aperture, non-linear dispersion, tune shifts, etc... One stops increasing the number of steps when these properties reach a limit. This is also the procedure to follow for a wiggler if you integrate the correct equation of motion with a symplectic integrator.

Unfortunately, we cannot find an explicit symplectic integrator for the Hamiltonian of Eq.(4). Therefore, one is forced to use a non-symplectic algorithm to integrate the motion explicitly. This is not criminal contrary to popular belief, especially in electron machine. The remainder of this section is devoted to the care and caution one must exercise when using a non-symplectic integrator. In general, the number of steps will depend strongly on the purpose of the study. Here are a few checks you should perform if you intend to use an ordinary integrator on this exact Hamiltonian. In general, speed is the problem. Relative to a symplectic integrator, you must use more steps in a nonsymplectic integrator to minimize the diseases caused by "non-symplecticity". 
Dynamic Aperture Calculation If you track without radiation, you should make sure that the damping or anti-damping of the integrator is sufficiently small. This will require some trial and error. The result will depend on the number of turns tracked.

If you track with radiation, then make sure that the actual damping decrement is correctly predicted by your integrator. If not, increase the number of steps. This will not depend significantly on the number of turns tracked.

Beam Envelope Calculation As in the radiative dynamic aperture, the beam sizes and the damping decrement must stabilize, therefore increase the number of steps until they do.

Taylor Series Map You can always produce a Taylor series map[6] for the wiggler. Since the Taylor series can be "symplectified" to the order of truncation (Dragt-Finn factorization)[7] or to all order (Irwin factorization)[8], the issue is whether or not your wiggler, in your machine, with its special parameters, can be represented by a Taylor series of finite degree. If the answer is yes, and the order of the Taylor map is low, this can be a good way to do fast tracking (always double check on a few cases with the painful nonsymplectic integrator).

Conclusion on the Exact Hamiltonian Integrating a wiggler by brute force and extracting maps out of the integrator is trivial. Remember that the work of Lloyd Smith[3], whose purpose was purely theoretical, is totally, completely irrelevant to the issue of particle tracking. Now, the question remains, can we do better (i.e. explicit symplectic integration) if we are willing to approximate $H$ slightly. This is the topic of the next section.

\section{Symplectic Methods on the Approximate Hamil- tonian}

In large rings, it is possible to expand the square-root Hamiltonian to leading order in $x^{\prime}$ and $y^{\prime}$. If applied to Eq.(2), the result is

$$
H=\frac{\left(p_{x}-f\right)^{2}}{2\left(1+p_{-\ell}\right)}+\frac{\left(p_{y}-g\right)^{2}}{2\left(1+p_{-\ell}\right)}+(1-\epsilon) p_{-\ell}
$$

For this Hamiltonian, it is possible to write immediately a first order explicit integrator by means of a generating function which we invert exactly. Consider the generating function $F$ :

$$
\begin{array}{ccc}
F(\boldsymbol{q}, \overline{\boldsymbol{p}}) & = & \boldsymbol{q} \cdot \overline{\boldsymbol{p}}+\Delta z H(\boldsymbol{q}, \overline{\boldsymbol{p}}) \\
\boldsymbol{q} & = & (x, y,-\ell) \\
\boldsymbol{p} & = & \left(p_{x}, p_{y}, p_{-\ell}\right) .
\end{array}
$$


The characteristic function $F$ generates the motion of any Hamiltonian $H$ for a step $\Delta z$ with a local error of order $(\Delta z)^{2}$. For the Hamiltonian of Eq.(7), this characteristic function produces equations which can be inverted exactly. The results are:

$$
\begin{gathered}
p_{i}=\frac{\partial F}{\partial q_{i}}=\bar{p}_{i}+\Delta z \frac{\partial H}{\partial q_{i}} \\
\bar{q}_{i}=\frac{\partial F}{\partial \bar{p}_{i}}=q_{i}+\Delta z \frac{\partial H}{\partial \bar{p}_{i}} \\
\bar{p}_{x}=\frac{\left\{p_{x}-\left(f f_{x}+g g_{x}\right) \Delta z_{p}\right\}\left(1-g_{y} \Delta z_{p}\right)+\left\{p_{y}-\left(f f_{y}+g g_{y}\right) \Delta z_{p}\right\} g_{x} \Delta z_{p}}{\left(1-f_{x} \Delta z_{p}\right)\left(1-g_{y} \Delta z_{p}\right)-f_{y} g_{x} \Delta z_{p}{ }^{2}} \\
\bar{p}_{y}=\frac{\left\{p_{y}-\left(f f_{y}+g g_{y}\right) \Delta z_{p}\right\}\left(1-f_{x} \Delta z_{p}\right)+\left\{p_{x}-\left(f f_{x}+g g_{x}\right) \Delta z_{p}\right\} f_{y} \Delta z_{p}}{\left(1-f_{x} \Delta z_{p}\right)\left(1-g_{y} \Delta z_{p}\right)-f_{y} g_{x} \Delta z_{p}{ }^{2}} \\
\bar{p}_{-\ell}=p_{-\ell}=x+\Delta z_{p}\left(\bar{p}_{x}-f\right) \\
\bar{x}=\sin \left(\bar{p}_{y}-g\right) \\
\bar{y}=\frac{\Delta z}{2}\left\{\left(\frac{\bar{p}_{x}-f}{1+p_{-\ell}}\right)^{2}+\left(\frac{\bar{p}_{y}-g}{1+p_{-\ell}}\right)^{2}\right\} \\
(-\bar{\ell})=(-\ell)-\frac{\Delta z}{1+p_{-\ell}}
\end{gathered}
$$

The expression of Eq.(9) is exactly symplectic for all possible functions $f$ or $g$. Therefore it is applicable to arbitrary wigglers and undulators. The deterministic radiation is calculated as in 2.1 by adding the vector $\Delta z \mathbf{r}$ to Eq.(9).

It is possible, with some degree of pain, to derive higher order explicit integrators, using the generating function used here and the theory of explicit symplectic according to Yoshida[9]. For the purpose of our discussion, this first order integrator will suffice.

\section{Discussion}

\subsection{Example}

In the case of symplectic integration, the actual accuracy is never a real issue. For example, we do rot cut the sextupoles into an increasing large number of thin lenses until convergence is reached. Instead we look at the global properties of the ring we are trying to estimate (dynamic aperture, geometric tune shifts, etc...), and we stop splitting the sextupoles when the properties reach approximately a limit. Quite often, sextupoles under such conditions, require only one thin lens per magnet. But, this is not a rule: for example, in a non-interleaved scheme the dynamic aperture of the bare machine (no fringe field) for the Hamiltonian of Eq.(7) becomes infinite with one thin lens per sextupole. That is 


\begin{tabular}{|c|ccc|}
\hline number of slices & 35 & 70 & 700 \\
\hline$\Delta \nu_{x}$ & -0.002567818 & -0.002573105 & -0.002579113 \\
$\Delta \nu_{y}$ & 0.007963092 & 0.007966845 & 0.007965440 \\
\hline
\end{tabular}

Table 1: Relation between the tune shifts and the integration step.

an infinitely incorrect answer, therefore one must use more steps per sextupoles. In the case of Eq.(7), we have an explicit (simple and fast) symplectic integrator which implies that we should treat the wiggler exactly as we treat multipoles.

For example, in the KEK photon factory, we simulated a helical wiggler with the following parameters:

$$
\begin{gathered}
F_{0}=0.12 m^{-1} G_{0}=0.024 m^{-1}, L=3.0 m, N_{\text {period }}=20 \\
k=\frac{2 \pi N_{\text {period }}}{L} m^{-1}, k_{1}=20.0 m^{-1}, k_{4}=20.0 m^{-1} \\
\nu_{x}=8.38, \nu_{y}=3.14 .
\end{gathered}
$$

We show the ( $x$ versus $p x$ ) and ( $y$ versus py) phase plots for 35 integration steps and 70 integration steps in figures 1 . Notice that 35 integration steps corresponds to an apparently outrageous 1.75 steps per period! For reference, the machine without wiggler is also shown in figures $1(\mathrm{a})$ and (b). Notice that the $\mathrm{x}$-px plots show clearly that the wiggler is not totally insignificant in this example.

Since the wiggler has a quadrupole component, we should take care of tune shifts. Table 1 shows a relation between the tune shifts and the integration steps. The differences between each integration steps are not significant.

Figures 2 are to be contrasted with a nonsymplectic integrator using a total of 120 function evaluations to go through the wiggler (instead of 35 and 70). So, as we said in section 2.2 , one must exercise extreme caution with nonsymplectic integrators as is already well-known. ${ }^{1}$

Also, we computed the dynamic apertures (on-momentum, 400 turns) for the 35 and 70 steps cases. Since the wiggler did not produce a significant difference from bare lattice, we also did double the wiggler strength to increase its effect. We also compute the bare machine aperture for reference. The results are displayed in figure 3 . This is not intended as a serious KEK photon factory study. It is an illustration of how to choose the number of steps in an integrator. (When one fixes the number of steps for sextupoles, one does not do it with serious dynamic aperture studies!)

\subsection{Conclusion}

They are two important points made in this paper:

\footnotetext{
${ }^{1}$ The reader should take our example with a grain of salt: we could be more clever in selecting a better behaved nonsymplectic integrator. For example, a first order nonsymplectic integrator which preserves the symplecticity of the linear part would do much better than this 6th order Runge-Kutta.
} 
1. Integrate in cartesian coordinate. It is the best choice to integrate wigglers.

2. If you can write a symplectic integrator, treat the wiggler like any other element. If you cannot, be very careful in tracking it (nonsymplectic integrator, Taylor series map, etc...).

Ackowledgment We would like to thank Dr.K.Hirata for beneficial discussions. One of the authors (E.F.) acknowledges the Foundation for High Energy Accelerator Science for financial support and Dr. S.Kurokawa for his gracious hospitality. He also acknowledges financial support from the United States Department of Energy under Contract No. DEAC03-76SF00098.

\section{References}

[1] K.Halbach, NIM.187 109 (1981).

[2] L.Tosi and A.Wrulich, ST/M-88/12.

R.Nagaoka and L.Tosi, ST/M-90/6.

[3] L.Smith,ESG TECH. NOTE-24.

[4] E.Forest and K. Hirata, A Contemporary Guide to Beam Dynamics, KEK report (1992).

[5] M.Sands, SLAC-121(1970).

[6] M.Berz,E.Forest and J.Irwin, SSC-166 (1988).

[7] A.J.Dragt and J.M.Finn, J.Math.Phys.20 2649 (1979).

[8] J.Irwin, SSC-228 (1989).

[9] H.Yoshida, Phys.Lett.A150 262 (1990).

E.Forest, J.Bengtsson and M.F.Reusch, Phys.Lett.A158 99 (1991). 

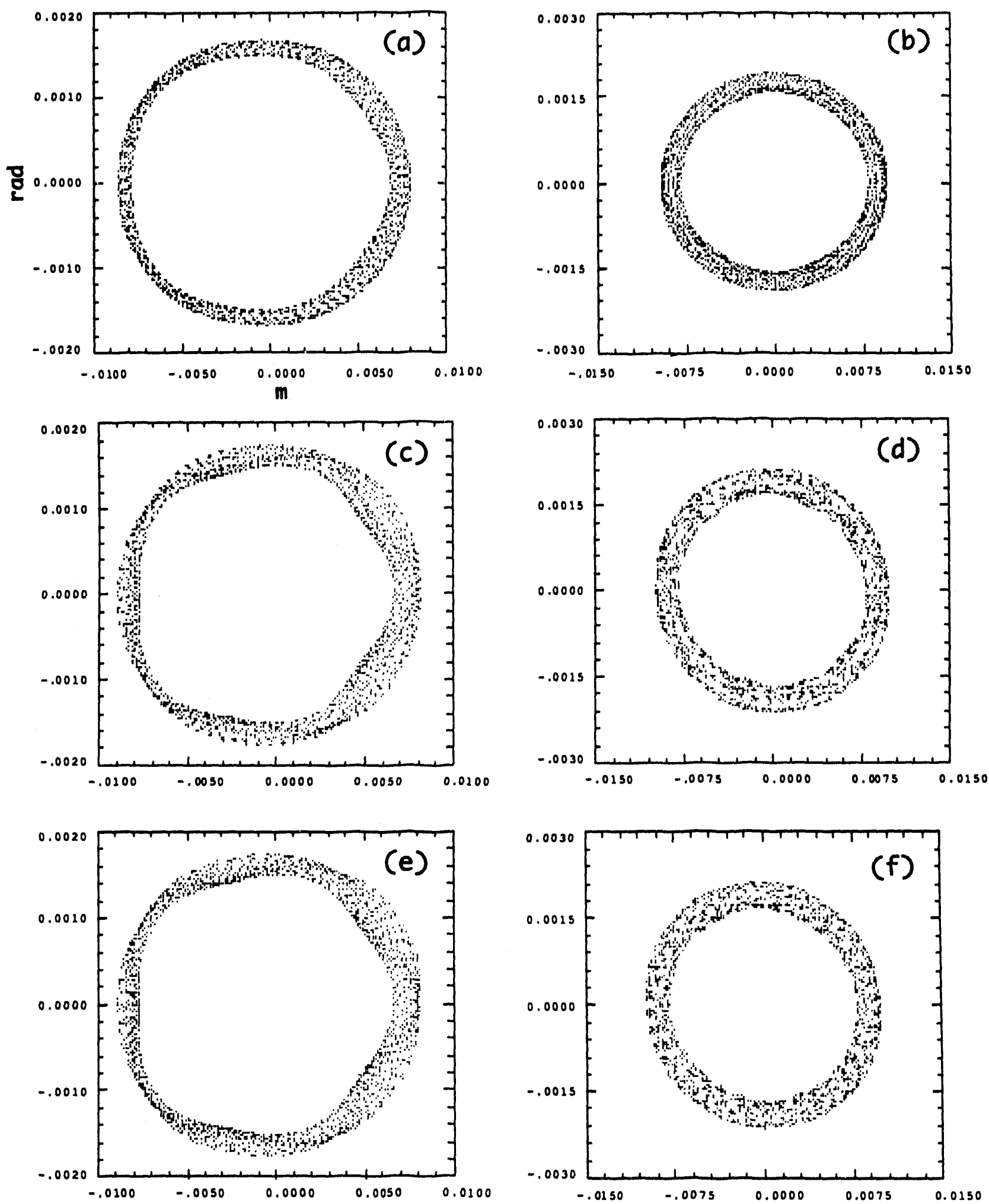

Figure 1: Phase space plots with 1st order symplectic integrator. Photon Factory with the wiggler is simulated. (a) and (b) are $x-p_{x}$ and $y-p_{y}$ plot without wiggler, respectively. (c) and (d) are for 35 integration steps. (e) and (f) are for 70 steps. 

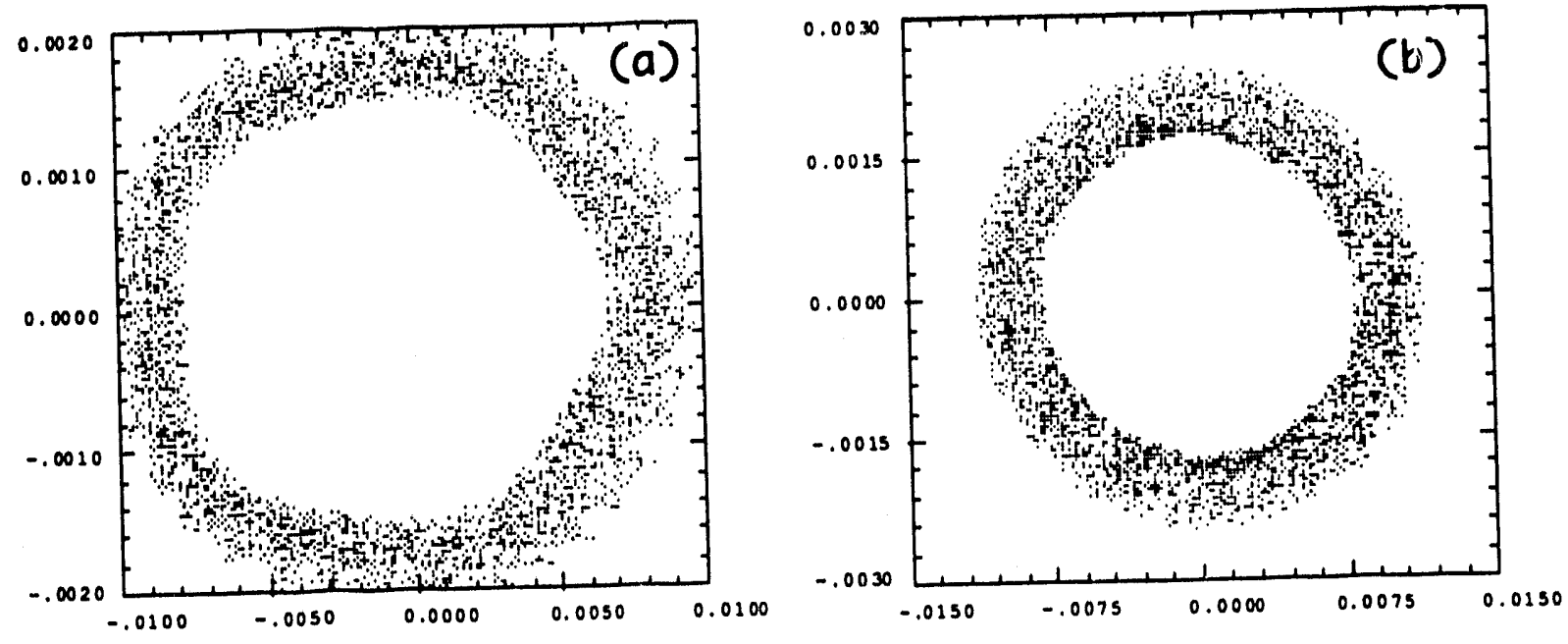

Figure 2: Phase space plots with 6-th order nonsymplectic Runge-Kutta integrator. (a) and (b) are $x-p_{x}$ and $y-p_{y}$ plot for 15 integration steps which is equivalent to 120 function evaluation.

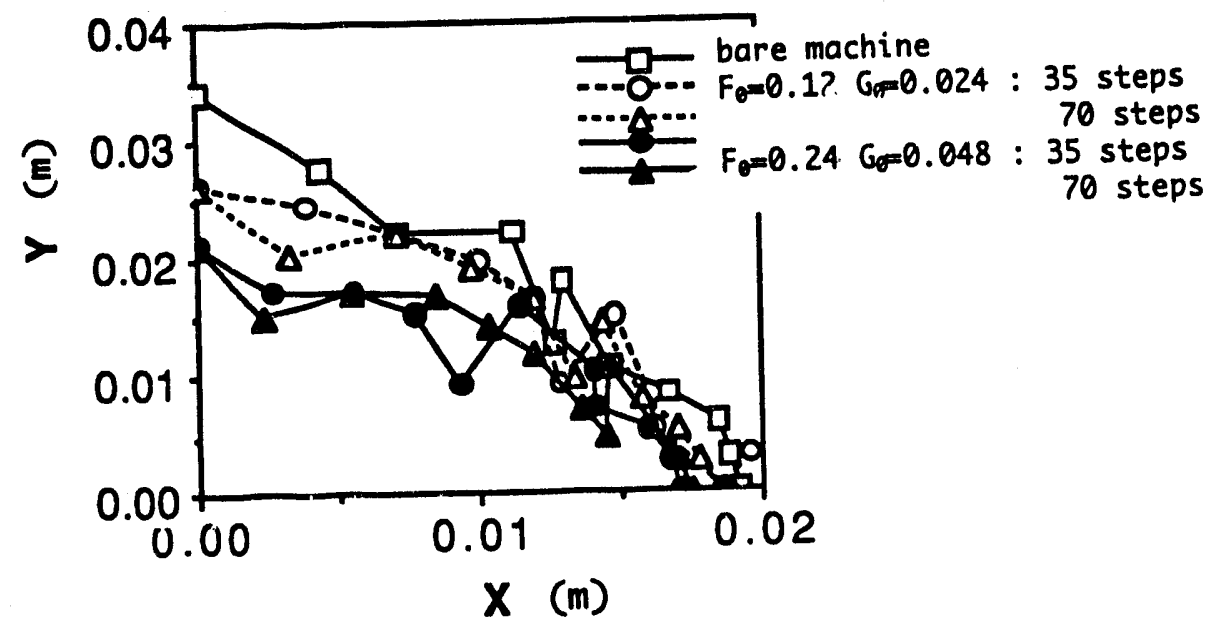

Figure 3: Dynamic aperture of $\mathrm{PF}$ ring with the wiggler. 

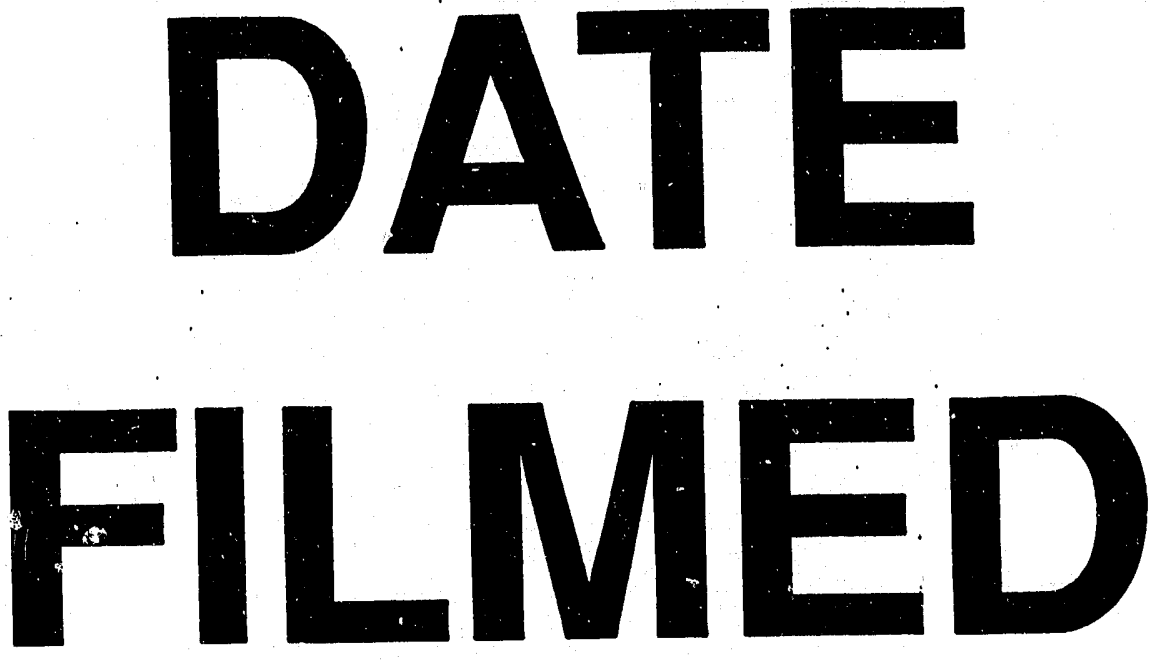

$8 / 17 / 93$
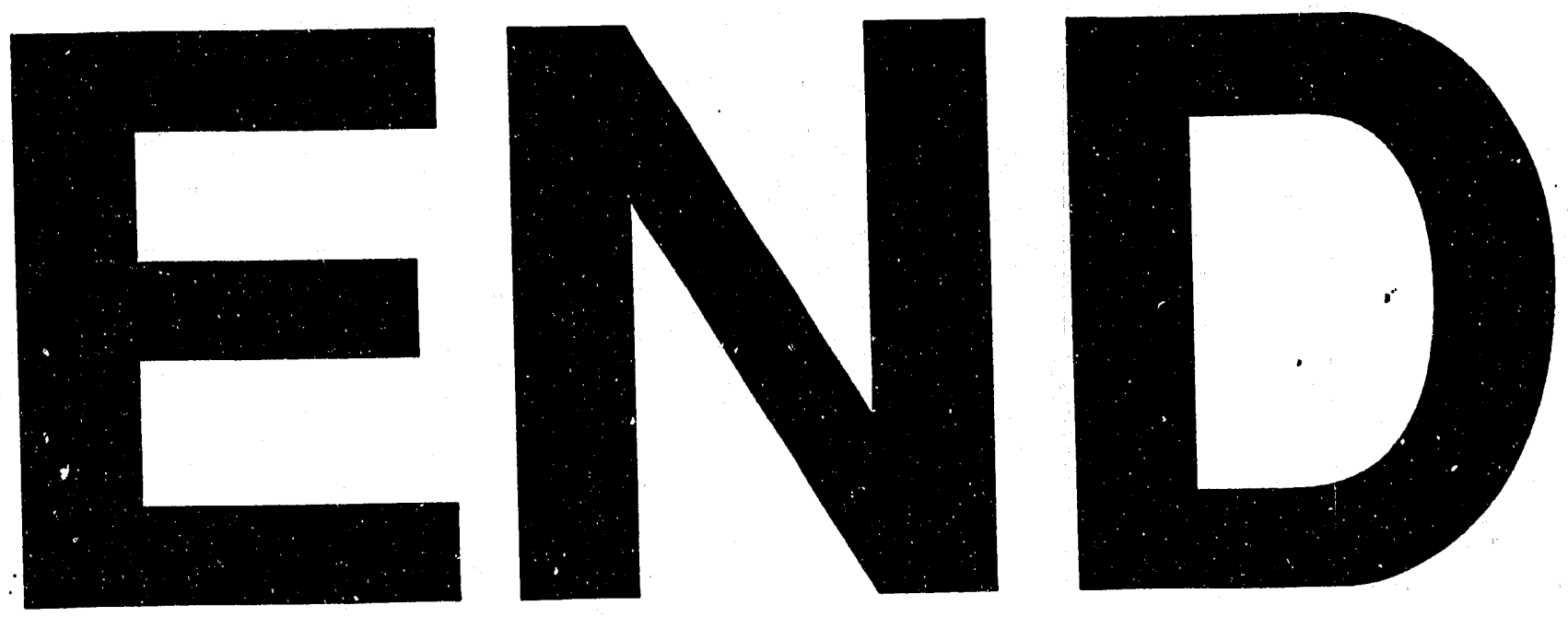
